\title{
Theoretical noise analysis on a position-sensitive Metallic Magnetic
}

\section{Calorimeter}

\author{
Stephen J. Smith \\ NASA Postdoctoral Program Fellow, NASA Goddard Space Flight Center, Code 662, Greenbelt, MD 20771, USA
}

\begin{abstract}
We report on the theoretical noise analysis for a position-sensitive Metallic Magnetic Calorimter (MMC), consisting of MMC read-out at both ends of a large X-ray absorber. Such devices are under consideration as alternatives to other cryogenic technologies for future X-ray astronomy missions. We use a finite-element model (FEM) to numerically calculate the signal and noise response at the detector outputs and investigate the correlations between the noise measured at each MMC coupled by the absorber. We then calculate, using the optimal filter concept, the theoretical energy and position resolution across the detector and discuss the trade-offs involved in optimising the detector design for energy resolution, position resolution and count rate. The results show, theoretically, the position-sensitive MMC concept offers impressive spectral and spatial resolving capabilities compared to pixel arrays and similar position-sensitive cryogenic technologies using Transition Edge Sensor (TES) read-out.
\end{abstract}

Keywords: Metallic Magnetic Calorimeter; position-sensitive X-ray detector; finite-element model; DROID; optimal filter; microcalorimeter

PACS: 95.55.Ka; 07.85.Fv; 29.40.Vj; 29.40.Gx

\section{$1.0 \quad$ Introduction}

Cryogenically cooled detectors such as Transition Edge Sensors [1], Metallic Magnetic Calorimeters [2] and Superconducting Tunnel Junctions [3], are under development by numerous groups worldwide for applications in X-ray astronomy. Large format pixel arrays (typically $32 \times 32$ ) [4] are required for future

\footnotetext{
· Corresponding author Tel.: 1-301-286-3719; fax: 1-301-286-1684; e-mail: sjs@milkyway.gsfc.nasa.gov
} 
astronomy missions such as NASA's Constellation-X [5]. The goal is to achieve full width at half maximum (FWHM) energy resolution of a few $\mathrm{eV}$ in the soft X-ray energy range.

Large absorber devices, using microcalorimeter or STJ read-out at both ends of a linear or segmented absorber [6-8], offer alternatives in achieving wide field coverage compared to pixel arrays. In the TES Distributed Read-Out Imaging Device (DROID), the sum of the two TES signals gives the energy of the absorbed photon and the difference between the signals gives the position information. Further details on these devices are reported elsewhere [8-10]. The development of such detectors is driven by the desire to achieve wide field coverage with a reduced number of read-out channels compared to the pixel arrays, thus providing a technologically simpler alternative and, the capability to extend the focal plane area for missions such as Constellation-X.

The position-sensing concept used in the TES DROID can, in principle, be implemented using MMC technology. In this paper we examine the theoretical performance of the MMC DROID concept.

An MMC consists of an X-ray absorber thermally coupled to a material whose magnetisation obeys a $1 / T$ Curie law dependence, at temperatures of the order $50 \mathrm{mK}$. The absorption of energy $\delta E$ in a detector with volume $V$ and heat capacity $C$ causes a change in magnetic moment $\delta m$, proportional to the deposited energy. The sensor is magnetised by a small B-field typically of the order a few mT. Changes in magnetic flux caused by X-ray absorption are measured using a SQUID (Superconducting Quantum Interference Device) magnetometer, which is directly flux coupled, or transformer coupled, to the sample. The magnetisation in MMCs comes from a dilute concentration of paramagnetic ions. This dilute concentration of randomly distributed ions in a host material is essential to limit the spin-spin interaction between ions, which result in a decrease in sensitivity. Unlike a dissipative thermistor such as a TES, MMCs require no bias power therefore dissipate no heat into the detector substrate and require no active bias circuitry. Thus the MMC is not subject to the same electrical and thermal design constraints as a thermistor and potentially, large format array development is technologically less challenging. Early results in single pixel MMCs have already 
demonstrated comparable resolution to other calorimeter types, $\Delta E=3.4 \mathrm{eV} \mathrm{FWHM} \mathrm{[2]} \mathrm{for} 5.9 \mathrm{keV}$ X-rays. Yet further improvements in resolution are theoretically predicted for fully optimised devices. MMCs therefore offer an exciting prospect for focal plane X-ray detectors.

\subsection{Detector signal and noise response}

We have established a linear finite-element model (FEM) to estimate the signal and noise response of the MMC DROID concept, similar to those described in refs. [11,12] for position-sensitive TES detectors. In the FEM, the MMCs are represented as each having two subsystems. Firstly, the electronic system of the MMC, including the embedded paramagnetic ions and the host absorber material, with a heat capacity $C_{e}$ and temperature $T_{e}$. Secondly, the MMC spin system with heat capacity $C_{z}$ and temperature $T_{z}$. The thermal conductance between the spin system and the electron system is $G_{z}$ and the thermal conductance between the electronic system and the bath, at temperature $T_{b}$, is $G$. The electron system is then connected to an absorber of length $L$, consisting of a series of $N$ (typically $N>100$ ) absorber heat capacities with overall thermal conductance $G_{a b s}$ and heat capacity $C_{a b s .}$. Figure 1 shows a schematic diagram of the FEM representing the MMC detector.

The noise sources in the detector come about from thermodynamic fluctuations between the various subsystems. These are: thermal fluctuation noise between the MMC and bath $P_{n}$, thermal fluctuation noise in each conductive element between absorber nodes $P_{n A b s}$ and thermal fluctuation noise between the MMC spin system and the electron system $P_{z}$. All these sources of noise have an intrinsic spectral density in the form $\sqrt{4 k_{b} T^{2} G}$ [13], where $k_{b}$ is Boltzmann's constant, $G$ is the thermal conductance between the relevant subsystems and $T$ is the temperature. The time evolution of the detector can then be represented by a series of coupled, linear, differential equations. Following the methodology of ref. [12] for a similar TES based DROID, this system of differential equations can be generalised as a single matrix equation and solved to find the signal and noise response at the detector outputs. The total noise, in terms of temperature 
fluctuations in the spin system, when the MMC signals are summed, can be written (in the frequency domain) as:

$\left\langle\left|N_{1+2}(f)\right|^{2}\right\rangle=\left\langle\left|N_{1}(f)\right|^{2}\right\rangle+\left\langle\left|N_{2}(f)\right|^{2}\right\rangle+2 \operatorname{Re}\left\langle N_{1} N_{2}^{*}(f)\right\rangle$,

where $\left\langle\left|N_{1}(f)\right|^{2}\right\rangle$ and $\left\langle\left|N_{2}(f)\right|^{2}\right\rangle$ are the noise powers in each MMC spin system and $\left\langle\left|N_{1} N_{2}^{*}(f)\right|\right\rangle$ is the noise covariance, which arises due to the coupling of the sensors via the absorber.

In this analysis we consider a simple geometry consisting of a MMC detector with a circular pickup coil-up, which is then transformer coupled to a SQUID. We note that different flux coupling geometries are also possible and yield slightly different optimisations conditions [14].

Considering a simple system of non-interacting spins, the flux change $\delta \Phi$ in a circular pickup loop of radius $r$, due to a change in temperature $\delta T$ is given by:

$\delta \Phi=\mu_{0} \frac{G_{k}}{r} V \frac{\partial M}{\partial T} \delta T$

where $G_{k}$ is a dimensionless factor which depends on the geometry between the spins and the pickup loop. The change in magnetisation with temperature $\partial M / \partial T$, is given by the thermodynamic expression [15]:

$\frac{\partial M}{\partial T}=\frac{C_{z}}{V} \frac{1}{B}$

where $V$ is the sensor volume and $B$ is the applied magnetic field. In reality the situation is more complex and an exchange interaction will exist between the spins. The existence of - Ruderman Kittel Kasuya Yosida (RKKY) [16] - exchange interactions will have a detrimental effect on MMC performance by reducing 
$\partial M / \partial T$ and hence, the device sensitivity [15]. In this régime: $\partial M / \partial T \propto x g^{2} B T^{2}$, where $x$ is the paramagnetic ion concentration, $g$ is the $g$-factor of the spins, $B$ is the magnetic field and $T$ is the temperature. A large $\partial M / \partial T$ is essential to ensure that the performance of the device is not limited by the flux noise from the SQUID. This requires careful optimisation of the device design and operating parameters, such as $x$ and $B$, to optimise the MMC resolution for a particular flux coupling scheme. Assuming a transformer coupled MMC, the value of $\partial M / \partial T$ to optimise the energy sensitivity, is numerically shown to be proportional to $g^{-1} \alpha^{-1}$, where $\alpha$ is the magnitude to RKKY exchange interactions (for a typical Er:Au system $g=6.8$ and $\alpha=5$ ) $[16]$.

Using Equation 2 the intrinsic detector noise can be converted into a flux noise in the pick-up coil:

$$
\begin{aligned}
\left\langle\left|\Phi_{M M C}\right|^{2}\right\rangle & =\left(\frac{\delta \Phi}{\partial T}\right)^{2}\left[\left\langle\left|N_{1}(f)\right|^{2}\right\rangle+\left\langle\left|N_{2}(f)\right|^{2}\right\rangle+2 \operatorname{Re}\left\langle N_{1} N_{2}^{*}(f)\right\rangle\right] \\
& =\left\langle\left|\Phi_{n 1}(f)\right|^{2}\right\rangle+\left\langle\left|\Phi_{n 2}(f)\right|^{2}\right\rangle+2 \operatorname{Re}\left\langle\Phi_{n 1} \Phi_{n 2}^{*}(f)\right\rangle .
\end{aligned}
$$

In addition to the intrinsic detector noise we must also consider noise from the SQUID read-out chain. We assume the pickup coil is flux coupled to the SQUID, therefore changes in flux in the pickup coil $\delta \Phi_{\text {ext }}$ generate a change in flux in the SQUID $\delta \Phi_{\mathrm{s}}$ given by:

$$
\delta \Phi_{s}=\frac{M_{i s}}{L_{p}+L_{i}} \delta \Phi_{e x t}=\frac{k \sqrt{L_{s} L_{i}}}{L+L_{i}}
$$

where $L_{p}$ is the inductance of the pickup coil, $L_{i}$ is the inductance of the input coil and $k$ is a coupling constant close to unity, $M_{i s}$ is the mutual inductance between the SQUID and the input coil. Thus the flux noise referred to the pickup coil is: 


$$
\left\langle\left|\Phi_{n s}\right|^{2}\right\rangle=\left\langle\left|\Phi_{s}\right|^{2}\right\rangle\left(\frac{\delta \Phi_{e x t}}{\delta \Phi_{s}}\right)^{2} .
$$

Using typical SQUID parameters $\left(L_{i}=L_{p}=50 \mathrm{pH}\right.$ and $\left.L_{s}=30 \mathrm{pH}\right)$, and assuming an intrinsic SQUID noise of $\Phi_{s} \approx 0.2 \mu \Phi_{0} / \sqrt{ } \mathrm{Hz}$, we calculate a flux noise in the pickup coil of $\Phi_{n s} \approx 0.5 \mu \Phi_{0} / \sqrt{ } \mathrm{Hz}$.

Magnetic Johnson noise will also be a contributing factor [15]. Magnetic Johnson noise is a flux noise caused by the thermal motion of charge carriers in the sensor, the absorber and other metallic materials in close proximity to the sensor. Using the data from ref. [14] for a typical Er doped Au sensor, the magnetic Johnson noise $\Phi_{J M M C}$ will be $\sim 0.1 \mu \Phi_{0} / \sqrt{ } \mathrm{Hz}$. The metallic absorber is also a potential source of magnetic Johnson noise. Again, from ref. [13] the flux noise $\Phi_{\text {Jabs }}$ is estimated to be $\sim 0.2 \mu \Phi_{0} / \sqrt{ } \mathrm{Hz}$.

The noise from the two SQUIDs and sources of magnetic Johnson noise will add incoherently with the detector noise, therefore, the total noise is:

$$
\left\langle\left|\Phi_{n t o t}\right|^{2}\right\rangle=\left\langle\left|\Phi_{M M C}(f)\right|^{2}\right\rangle+2\left\langle\left|\Phi_{n s}\right|^{2}\right\rangle+2\left\langle\left|\Phi_{J M M C}\right|^{2}\right\rangle+2\left\langle\left|\Phi_{\text {Jabs }}\right|^{2}\right\rangle
$$

Figure 2 shows, using the device parameters in Table 1, the calculated flux noise spectral density in the pickup loop for one of the MMCs, $\Phi_{n 1}$. Figure 3 then shows the total noise spectral density $\Phi_{M M C}$ when the signals from both MMCs are summed together. To examine the intrinsic detector limits we set the magnetic Johnson noise and the SQUID noise initially to zero.

Modelling of the noise spectrum reveals the frequency dependent correlations between the individual noise sources when sampled on each MMC. This is an identical situation to TES DROID except we have different sources of detector noise [10]. Both MMCs will sense all noise contributions via the coupling of the 
absorber; however, one MMC will sense noise attenuated and phase shifted relative to the other. When the two signals are summed, a frequency dependent correlation will exist. This is most clearly indicated in the absorber phonon noise, where at low frequency, the noise contributions tend to cancel. These correlations are quantified from the covariance $\left\langle\Phi_{1} \Phi_{2}{ }^{*}(f)\right\rangle$ of the data (see Figure 4).

The phonon noise in the absorber is heat moving between adjacent heat capacities. At low frequency one MMC will detect a drop in temperature while the other detects an increase, thus when the signals are added, the noise effectively cancels. As the frequency is increased, a change in phase will be observed at the MMCs and the correlation changes.

The phonon fluctuation noise between the MMC and the bath shows the opposite. At low frequency a positive correlation arises because the absorber has time to equilibrate and the phonon bath noise measured at MMC 1 will be sensed on MMC 2 in phase, so when combined the two contributions add. The positive, in-band, correlation of the phonon bath noise means that their contributions to the total summed noise are added linearly and not in quadrature. The phonon bath noise for a single pixel MMC with bath conductance $G$, will therefore be the same as a MMC DROID, with each MMC-bath conductance of G/2.

At low frequency, the thermal fluctuations between the spin-electron system from MMC 2, measured on MMC 1, tend towards zero. This is because, on long time scales the cold bath acts as a heat sink with infinite heat capacity; thus, in the zero frequency limit, noise fluctuations will flow to the heat bath and not be sensed by MMC 2. Consequently, at zero frequency there exists no correlation between the spin electron noise on each MMC. As the frequency is increased, more heat will flow through the absorber and a correlation arises.

\subsection{Energy and Position Resolution}


Under the régime of optimal filtering [17], the energy resolution of a microcalorimeter is calculated from the noise-equivalent-power (NEP) [18]. The energy resolution of a single pixel MMC, assuming just an electron and spin subsystem, can then be written as [14]:

$$
\Delta E_{F W H M} \approx 2.35 \sqrt{k_{b} T^{2} C_{e}}\left(\frac{1}{\beta(1-\beta)}\right)^{1 / 4}\left(\frac{\tau_{0}}{\tau_{1}}\right)^{1 / 4},
$$

and is limited by fluctuations in energy exchange between the spins and the electrons. Here, $\tau_{0}\left(\sim 10^{-6} \mathrm{~s}\right)$ is the relaxation time constant between the spins and the electrons and $\tau_{1}\left(\sim 10^{-4} \mathrm{~s}\right)$ is the thermal time constant between the detector and the heat sink. The parameter $\beta=C_{z} /\left(C_{z}+C_{e}\right)$, is the normalised heat capacity ratio. In the context of a canonical ensemble of thermal subsystems, as in our FEM, $\tau_{0}$ is defined by the thermal equilibrium time constant between the spin and electron system and thus depends explicitly on $C_{z}$ and $G_{z}$. However, in reality the thermal conductance $G_{z}$ between the spin system and electron system is not an adjustable parameter. The time constant $\tau_{0}$ is more appropriately defined by the intrinsic, material dependent, spin-electron relaxation time (which varies inversely with temperature [14]) and as such is independent of the parameters $C_{z}$ and $G_{z}$. Thus, for a single pixel MMC, with a fixed ratio $\tau_{0} / \tau_{1}$, Equation 8 is minimised when $C_{z}=C_{e}\left(\beta=\_\right)$. Given the complexity of the DROID system, the appropriate optimisation of heat capacities is less obvious. We can however attempt to put some limits on what the optimisation should be. Assuming a MMC DROID with a short absorber thermal equilibration time $\tau_{a b s}$ (sufficiently large $G_{a b s}$ ) compared to the thermal time constant between the MMC and heat sink $\tau_{1}$, the MMCs will be strongly thermally coupled to the absorber electron system, and our detector will behave more like a single MMC. Then, from Equation 8, it follows that the detector energy resolution will be optimised when $C_{z}=C_{e}+C_{a b s} / 2$. However, in reality, $G_{a b s}$ must be designed to give enough spatial variation in pulse shape across the absorber to enable position determination. If $\tau_{a b s}$ and $\tau_{1}$ are of similar time scales the thermal coupling of the MMCs to the absorber will be weaker and this optimisation may not be appropriate. In this régime, optimisation of the individual sensors, such that $C_{z}=C_{e}$, may be more applicable. 
Despite the limitations of our FEM in truly representing the physical nature of the MMC DROID, we can investigate the role thermal diffusion (determined by $G_{a b s}$ ) in the absorber has on energy and position resolution for a fixed set of MMC design parameters. The use of optimal filtering to numerically calculate energy and position resolution for position-sensitive TES DROIDs is described by ref. [12] and can be applied to the equivalent MMC concept, though we note these algorithms are yet to be implemented on real data. For this analysis we use the parameters in Table 1, which are estimates derived using the data in ref. [14], for optimised energy sensitivity of each individual sensor (assuming a typical single pixel Er doped Au $\mathrm{MMC})$.

For a TES based DROID, we have shown that the resolution depends explicitly on the ratio of the thermal conductances $G_{a b s} / G$, and is independent of their exact values (though the permissible count rate is not) [12]. However, only in the physically unrealistic limit $\tau_{0} \rightarrow 0$, will the MMC DROID depend only on $G_{a b s} / G$. Figure 5 shows the calculated energy resolution at the centre of the device, as a function of $G_{a b s}$, evaluated for different $G$ values, all other parameters are fixed. Only the intrinsic detector noise sources are included. Optimised energy resolution requires large $G_{a b s} / G$, that is, rapid thermal diffusion across the absorber. For a segmented absorber design (such as in [6]), the position resolution depends on the pixel pitch (typically 250 $\mu \mathrm{m}$ is adequate for most astrophysics applications). However, for a continuous absorber design, the theoretical position resolution can be calculated from the optimal filtering algorithms described in ref. [12]. Figure 6 shows the position resolution at the centre of the device and Figure 7 shows the trade-off between energy and position resolution for the different $G$ and $G_{a b s .}$. The position resolution is inversely proportional to incident photon energy and is calculated assuming a $1 \mathrm{keV}$ photon. The minimum in position resolution occurs because of the requirement to have enough variation in the pulse shape across the absorber to enable position determination, while maintaining optimum noise performance.

The error bars for the $G=1 \mathrm{nWK}^{-1}$ line in Figure 7, show the effect of including the SQUID flux noise and the magnetic Johnson noise. The effect on the energy resolution is less than $8 \%$ for all $G_{a b s}$. The effect on 
the position resolution is more significant, especially away from the minimum, where there is a $\sim 20 \%$ degradation in position resolution at $G_{a b s}=100 \mathrm{nW} \mathrm{K}$. These effects will be worse if the MMC sensitivity $(\partial M / \partial T)$ is reduced, thus, particularly for high position resolution applications, optimisation of the device sensitivity is essential.

A potential disadvantage of the large absorber MMC is in the count rate. The decay time of the pulse $\tau$ and hence the count rate, depends on the thermal conduction to the bath $G$. A MMC does not have the advantage of a reduced intrinsic $(C / G)$ time constant as for a TES detector operated with electrothermal feedback (where typically $\tau \sim 100 \mu \mathrm{s}$ ) [1]. Furthermore, the dependence of the time constants on resolution (Equation 8) means there is an intrinsic trade-off between energy resolution and count rate. For $G_{a b s} \geq G$, the pulse decay time for the MMC DROID is given by $\tau \approx C_{\text {tot }} / 2 G$, where $C_{\text {tot }}$ is the total heat capacity including both MMCs and the absorber. A fast decay time of $\sim 250 \mu \mathrm{s}$ is achievable for $G=10 \mathrm{nW} \mathrm{K} \mathrm{K}^{-1}$, and although the theoretical energy and position resolution are not optimised they still give eV energy resolution and $\mu \mathrm{m}$ position resolution in the $\mathrm{keV}$ energy range, comparable to the TES DROID. The advantages of the MMC DROID over the TES counterpart are likely to be related to more practical aspects. The reproducibility of the TES transition temperature and shape between sensors is not yet at the level required for the detector responses to be perfectly symmetric [19]. Such variations between sensors complicate the operation of the device and ultimately can result in a loss of resolution. Furthermore, non-linearity in the detector response and TES saturation at high photon energy, particularly for events absorbed close to the TESs [6], can also result in a loss of resolution. However, for the MMCs, the intrinsic reproducible, linearity of $\partial M / \partial T$ [15] means the detector response is much more linear with energy and the sensor responses should be symmetric. Furthermore, over the typical operating energy range the MMC will not suffer saturation at high photon energy as with a TES detector. These factors make the pursuit of a position-sensitive MMC an exciting prospect.

\subsection{Conclusions}


Using a finite-element model we have investigated the theoretic noise response of a position-sensitive, onedimensional imaging spectrometer based around MMC technology. We have numerically calculated the theoretical best achievable energy and position resolution, assuming optimal filtering, across a $2 \mathrm{~mm}$ device using typical MMC parameters from literature. The analysis reveals the trade-offs in designing such detectors for different applications and provides a framework for the development of a practical device, which would further extend the possibilities for cryogenic focal plane instruments in X-ray astronomy. The practical advantages such as reproducibility of the signal response between sensors means that ultimately the development of MMC DROIDs may offer a technologically simpler alternative to the TES DROID. We have also extended the analysis to investigate two-dimensional systems such as a square absorber with MMC readout in each corner. In general, the noise correlations between sensors and the trade-offs between energy and position resolution follow the same scaling as for the one-dimensional equivalent. However, for a given number of readout sensors per absorber pixel, the total heat capacity will be twice as big compared to the

one-dimensional case. Thus the energy resolution will be $2^{1 / 2}$ worse for an array of two dimensional DROIDs, compared an array of one dimensional DROIDs with the same number of readout channels. Consequently, the development of the two dimensional devices offers a less attractive alternative.

\section{Acknowledgements}

This research was supported in part by an appointment to the NASA Postdoctoral Program at Goddard Space Flight Center, administered by Oak Ridge Associated Universities through a contract with NASA. This work was part funded by the University of Leicester Space Research Centre, UK. The author would like to thank Dr. Simon Bandler and Dr Caroline Kilbourne of NASA Goddard Space Flight Center for useful discussions in the preparation of the manuscript. 


\section{References}

1. K.D. Irwin, G.C. Hilton, D.A. Wollman, J.M. Martinis, X-ray detection using a superconducting transitionedge sensor microcalorimeter with electrothermal feedback, Appl. Phys. Lett 69 (1996) 1945.

2. A. Fleischmann, M. Link, T. Daniyaro, H. Rotzinger, C. Enss, G. M. Seidel, Metallic magnetic calorimeters (MMC): detctors for high-resolution X-ray spectroscopy, Nucl. Instr. and Meth. A 520 (2004) 27.

3. D. Twerenbold, Superconducting tunnelling junctions as X-ray detectors and their possible applications in astrophysics, Nucl. Instr. and Meth. A 273 (1988) 575.

4. C.A. Kilbourne, S.R. Bandler, A.D. Brown, J.A. Chervenak, E. Figueroa-Feliciano, F. M. Finkbeiner, N. Iyomoto, R.L. Kelley, F.S. Porter, T. Saab, J. Sadleir, J. White, High-density arrays of x-ray microcalorimeters for Constellation-X, Proc. SPIE 6266 (2006) 59.

5. A.E. Hornschemeier, N.E. White, H. Tananbaum, The frontier in x-ray spectroscopy: NASA's ConstellationX mission, AIP Conf. Proc. 774 (2005) 383.

6. N. Iyomoto, S.R. Bandler, R.P. Brekosky, J. Chervenak, E. Figueroa-Feliciano, F.M. Finkbeiner, R.L. Kelley, C.A. Kilbourne, M.A. Lindeman, K. Murphy, S. Porter, T. Saab, J.E. Sadlier, D.J. Talley, Positionsensitive transition-edge sensors, Nucl. Instr. and Meth. A 559 (2006) 491.

7. R.A. Hijmering, P. Verhoeve, D.D.E. Martin, A. Peacock, A.G. Kozorezov, R. Venn, Imaging spectroscopy with Ta/Al DROIDs: Performance for different absorber lengths, Nucl. Instr. and Meth. A 559 (2006) 692.

8. S.J. Smith, C.H. Whitford, G.W. Fraser, D.J. Goldie, Characterisation and modelling of Transition Edge Sensor Distributed Read-Out Imaging Devices, Nucl. Instr. and Meth. A 559 (2006a), 500

9. S.J. Smith, C. Whitford, G.W. Fraser, A.D. Holland, D. Goldie, T.J.R Ashton, R.J. Limpenny, T. Stevenson, First results from a 1-D imaging spectrometer using Ir TESs, Nucl. Instr. and Meth. A 520 (2004) 449.

10. S.J. Smith, Development of Transition Edge Sensor Distributed Read-Out Imaging Devices for Applications in X-ray Astronomy, $\mathrm{PhD}$ Thesis, University of Leicester, 2006b.

11. E. Figueroa-Feliciano, Complex microcalorimeter models and their application to position-sensitive detectors, J. Appl. Phys. 99 (2006) 114513.

12. S.J. Smith, C.H. Whitford, G.W. Fraser, Optimised filtering for improved energy and position resolution in TES based X-ray detectors, Nucl. Instr. and Meth. A, 556 (2006c) 237. 
13. J.C. Mather, Bolometer noise: nonequilibrium theory, Applied Optics 21 (1982) 1125.

14. A. Fleischmann, C. Enss, G.M. Seidel, Metallic Magnetic Calorimeters, Cryogenic Particle Detection, Topics in Applied Physics 99 (2005), edited by C. Enns.

15. C. Enss, A. Fleischmann, K. Horst, J. Schonefeld, J. Sollner, J.S. Adams, Y.H. Huang, Y.H. Kim, G.M. Seidel, Metallic magnetic calorimeters for particle detection, J. Low Temp. Phys. 121 (2000) 137.

16. M.A. Ruderman, C. Kittel, Indirect Exchange Coupling of Nuclear Magnetic Moments by Conduction Electrons, Phys. Rev. 96 (1954) 99.

17. A.E. Szymkowiak, R.L. Kelley, S.H. Moseley, C.K. Stahle, Signal Processing for Microcalorimeters, J. L. Temp. Phys. 93 (1993) 281.

18. S.H. Moseley, J.C. Mather, D. McCammon, Thermal detectors as x-ray spectrometers, J. Appl. Phys. 46 (1984) 1257.

19. E. Figueroa-Feliciano, Position-sensitive transition edge sensors: recent progress and current issues, presented at the $3^{\text {rd }}$ International Workshop on Transition Edge Sensor Device Physics, University of Florida, August $17^{\text {th }}-19^{\text {th }} 2006$. 
Table 1. Parameters used to calculate the MMC DROID noise spectral density.

\begin{tabular}{|c|c|}
\hline Parameter & Value \\
\hline$C_{z}$ & $1 \mathrm{pJ} \mathrm{K}^{-1}$ \\
\hline$C_{e}$ & $1 \mathrm{pJ} \mathrm{K}^{-1}$ \\
\hline$C_{a b s}$ & $1 \mathrm{pJ} \mathrm{K}^{-1}$ \\
\hline$G_{a b s}$ & $25 \mathrm{nW} \mathrm{K}^{-1}$ \\
\hline$G_{z}$ & $500 \mathrm{nW} \mathrm{K}^{-1}$ \\
\hline$G$ & $1 \mathrm{nW} \mathrm{K}$ \\
\hline$L$ & $2 \mathrm{~mm}$ \\
\hline$\partial M / \partial T$ & 44342 \\
\hline$T$ & $50 \mathrm{mK}$ \\
\hline$V$ & $2050 \mu \mathrm{m}^{3}$ \\
\hline$r$ & $10.7 \mu \mathrm{m}$ \\
\hline$G_{k}$ & 0.5 \\
\hline
\end{tabular}




\section{Figure Captions}

Figure 1. Schematic diagram of the finite-element model used to calculate the response of the position-sensitive MMC concept. Only one MMC and part of the absorber is included.

Figure 2. Flux noise spectral density $\left(\mu \Phi_{0} / \sqrt{ } \mathrm{Hz}\right)$ in the pickup coil of $\mathrm{MMC} 1$ in a two MMC detector. Included is the phonon-bath noise (blue triangles) and spin-electron noise (red diamonds) associated with MMC 1, with spectra similar to that expected from a single pixel MMC [14]. The phonon-bath (purple crosses) and spinelectron noise (orange pluses) from MMC 2 are all coupled through the absorber and therefore appear on the MMC 1 read-out. The thick dashed line gives the total noise contribution from each absorber element. The thick solid line gives the total noise spectrum. The thin solid line is signal response from a $1 \mathrm{keV}$ photon absorbed at the centre of the device.

Figure 3. Flux noise spectral density $\left(\mu \Phi_{0} / \sqrt{ } \mathrm{Hz}\right)$ when both $\mathrm{MMC}$ data is summed. The key is the same as in Figure 1. Due to symmetry of the device, some contributions are overlaid.

Figure 4. Correlation coefficient, defined as the normalised covariance $\left\langle\Phi_{1} \Phi_{2}{ }^{*}(f)\right\rangle /\left\langle\left|\Phi_{\mathrm{n} 1}(f)\right|\right\rangle /\left\langle\left|\Phi_{\mathrm{n} 2}(f)\right|\right\rangle$, between the noise measured on the two MMCs. Included is the total correlation (black solid), phonon-bath (blue dashed), spin-electron noise (red dot-dashed).

Figure 5. Energy resolution vs. $G_{a b s}$ for four different values of $G$.

Figure 6. Position resolution vs. $G_{a b s}$ for four different values of $G$.

Figure 7. Trade-off between energy and position resolution for different $G$ and $G_{a b s}$. For the plot corresponding to $G=1 \mathrm{nW} / \mathrm{K}$, the error bars indicate the resolution including the contributions from the SQUID noise and magnetic Johnson noise. 


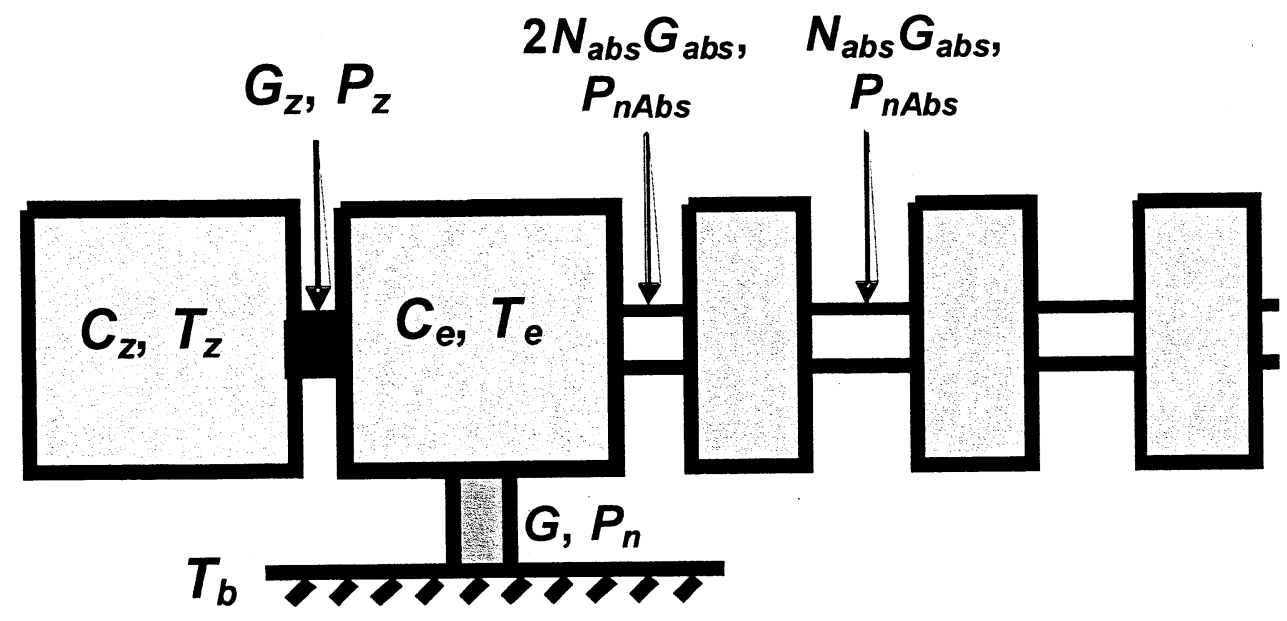

Figure 1 


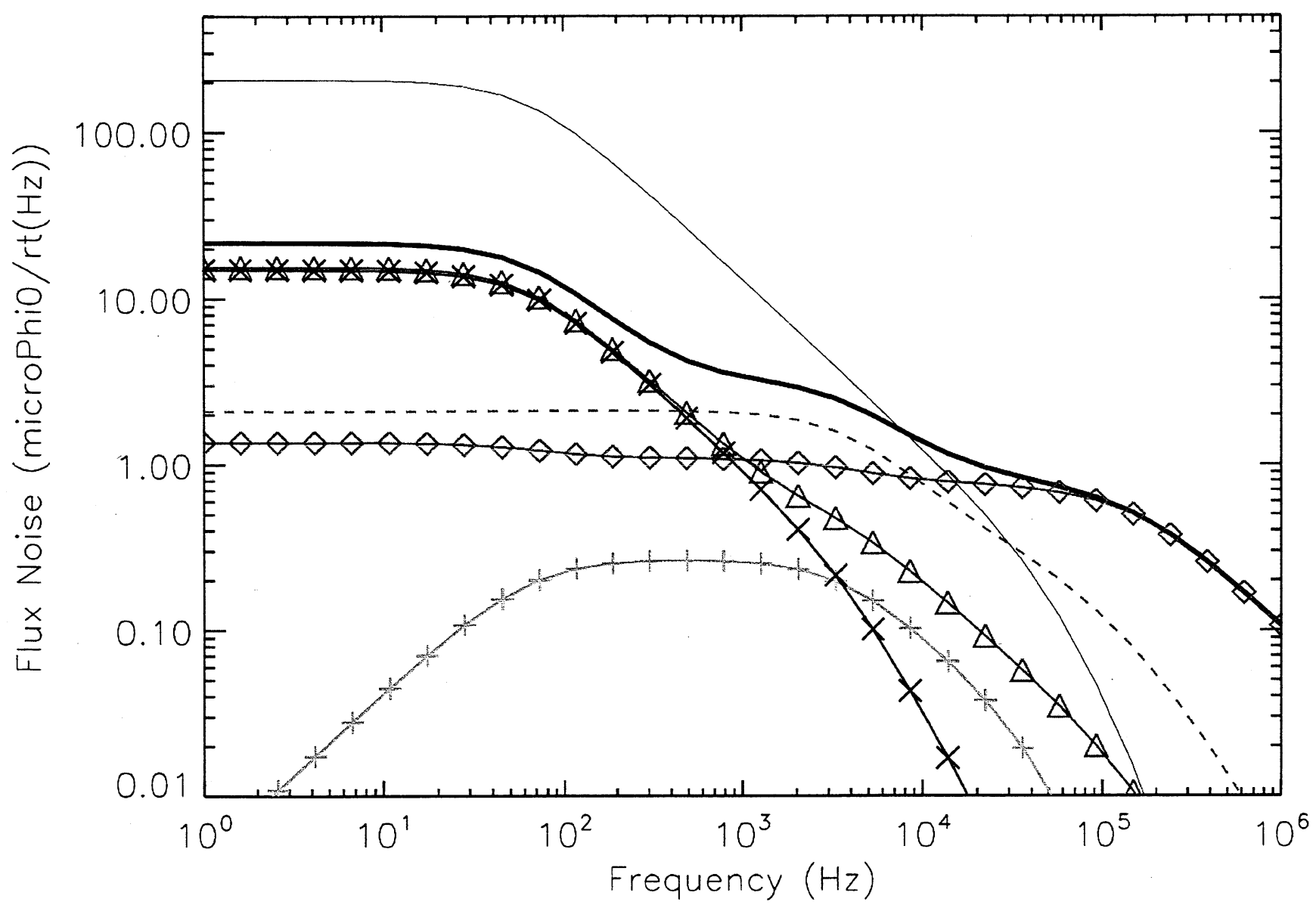

Figure 2 


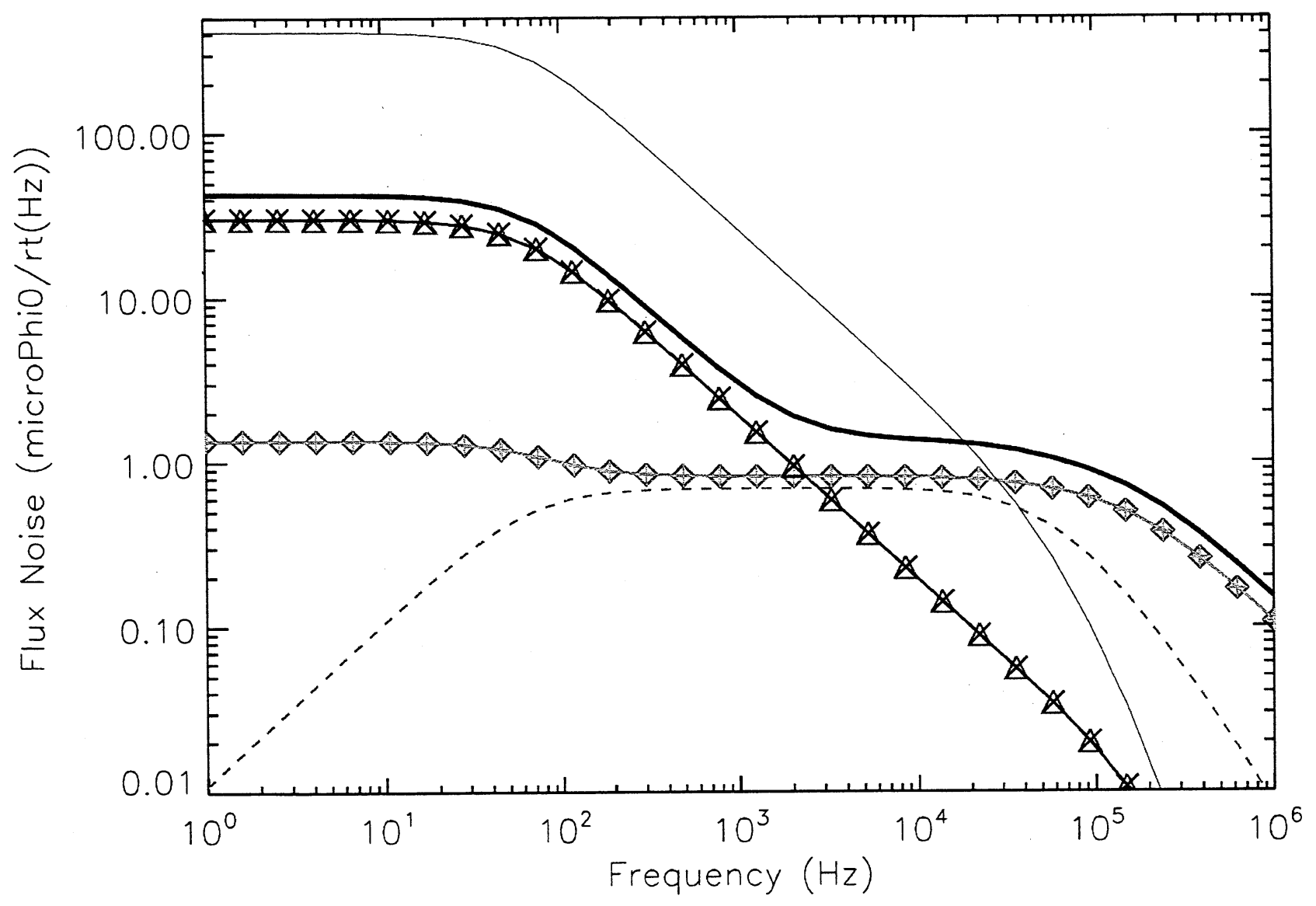

Figure 3 


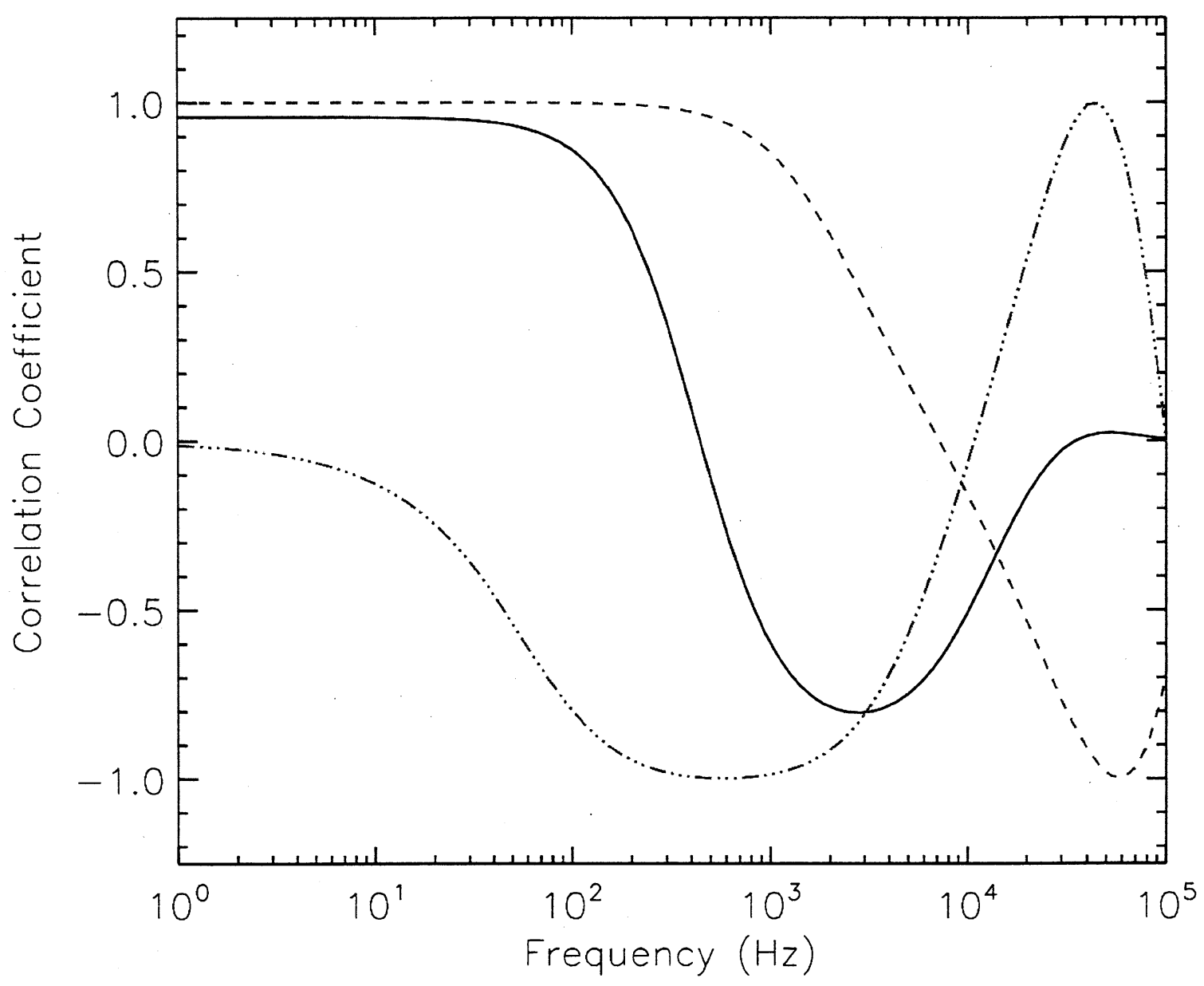

Figure 4 


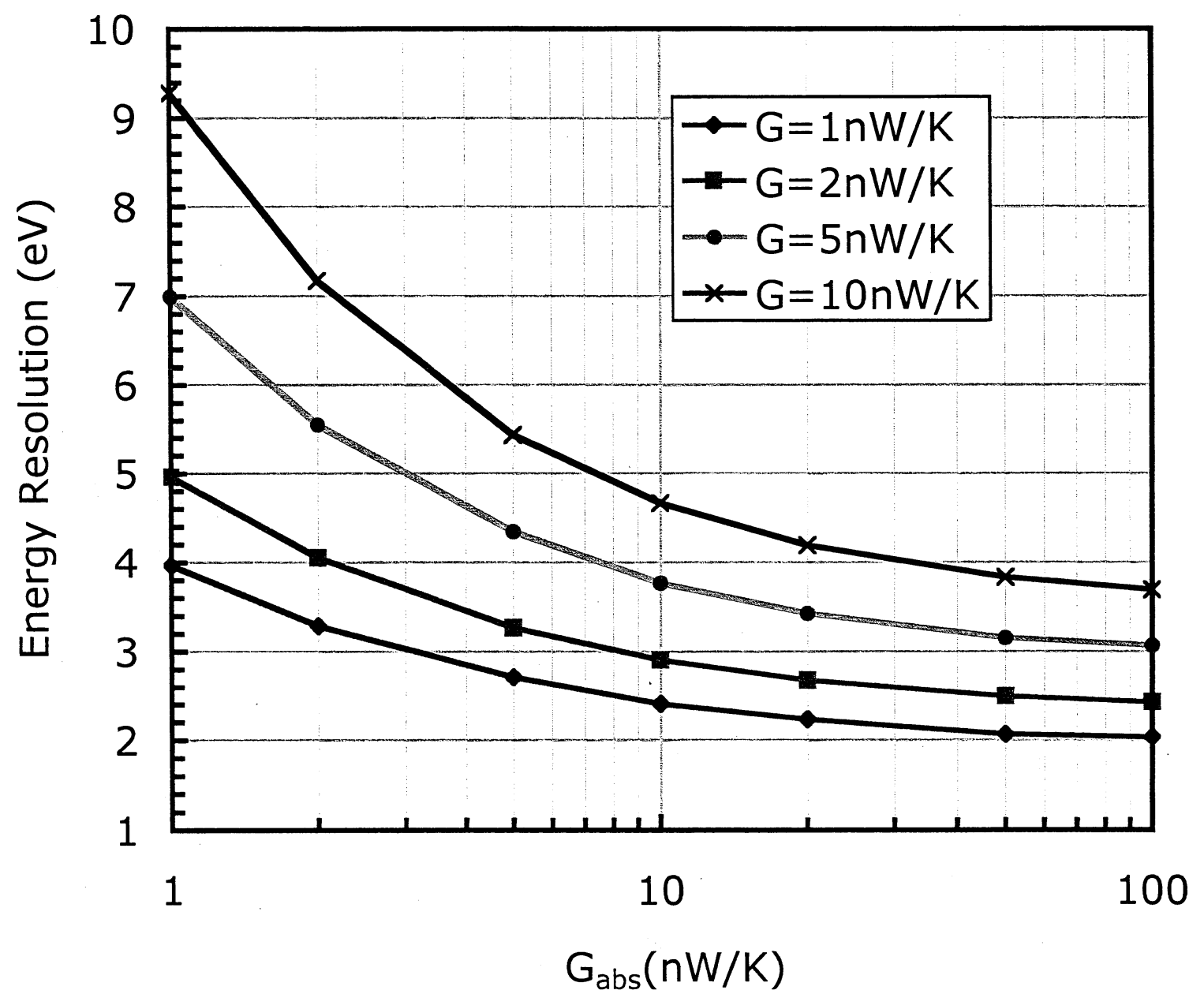

Figure 5 


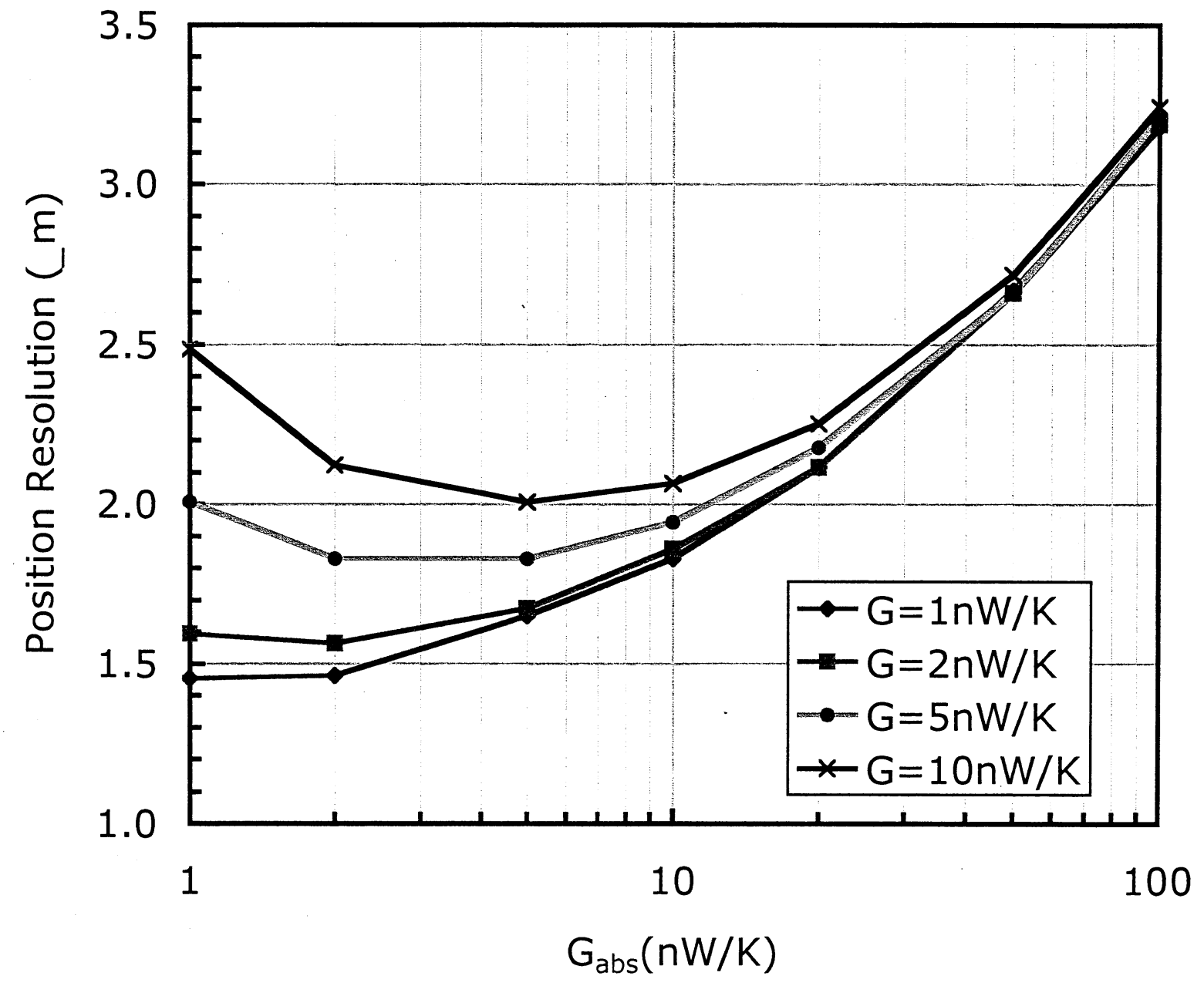

Figure 6 


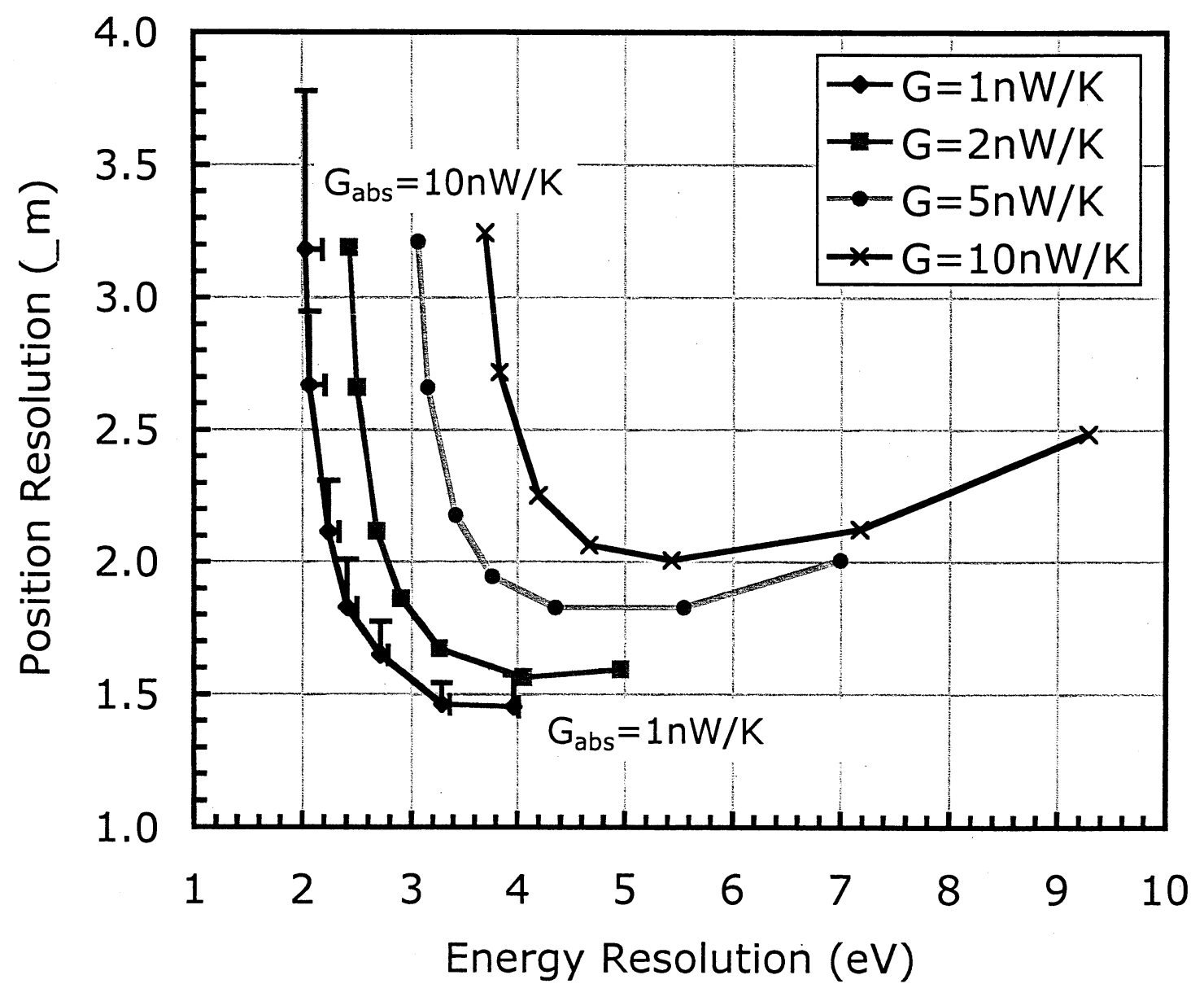

Figure 7 